\title{
The Soria Moria approach to pediatric forensic issues
}

\author{
Roger W. Byard $\cdot$ Henry F. Krous • \\ Torleiv O. Rognum
}

Accepted: 10 June 2013/Published online: 6 July 2013

(C) Springer Science+Business Media New York 2013

Pediatric forensic pathology has been a controversial and evolving area in recent years [1, 2]. High profile court cases in many countries, including the United Kingdom and Canada, have focused both professional and public attention on deficiencies in diagnoses and approaches to cases, sometimes with resultant highly unfortunate miscarriages of justice [3, 4]. Examples of irresponsible testimony in court by physicians in cases of child maltreatment and neglect, as well as the medicolegal consequences caused by a medical examiner's inadequate expertise and experience in pediatric pathology, have been described $[5,6]$. Attempts to redress this situation have included the involvement of professional organizations and courts in reviewing cases and in establishing protocols and guidelines. A special issue of Forensic Science Medicine and Pathology was published in 2010 in which a number of issues were reviewed and analyzed including terminology and classifications for unexpected deaths in infancy and the place of parents at infant death scenes [7-9]. Additional problems in the field include declining numbers of trainee

\footnotetext{
R. W. Byard ( $\square)$

Discipline of Anatomy and Pathology, The University of Adelaide, Level 3 Medical School North Building, Frome Road, Adelaide, SA 5005, Australia

e-mail: roger.byard@sa.gov.au

H. F. Krous

UCSD School of Medicine, La Jolla, CA, USA

H. F. Krous

Rady Children's Hospital, San Diego, CA, USA

T. O. Rognum

Department of Forensic Pathology and Clinical Forensic

Medicine, The Norwegian Institute of Public Health,

University of Oslo, Oslo, Norway
}

pathologists who are interested in pediatric forensic issues and adult practitioners who sometimes make the mistake of extrapolating from adult cases back to children. One way of addressing these problems is to hold meetings that specifically focus on pediatric forensic issues.

This has not, however, been a common occurrence. An exception to this is the annual meeting convened in Norway by the Department of Forensic Pathology and Clinical Forensic Medicine of the Norwegian Institute of Public Health at the Soria Moria Convention Center, outside the main city of Oslo. The meeting has been held on an annual basis since 1993; it is an international meeting with English as the official language, although often with a Norwegian meeting held on the preceding day. The meeting lasts for two and a half days and covers a wide range of topics focusing particularly on sudden infant death syndrome (SIDS) and on sudden and unexpected infant and childhood death. The meeting is quite small limited to between 40 and 60 participants, who come from a variety of forensic, clinical, and research backgrounds.

To give an insight into the types of presentations that occur, the program for this year (2013) will be reviewed. On the first morning, a session was held on the epidemiology of SIDS and sudden death in infants and children. This included overviews of changing patterns in mortality and trends in classifications, the relationship of SIDS rates to immunizations, death scene investigations in Norway, and discussion on parental responses to death scene examinations and bereavement issues. The morning concluded with a session where participants were able to have short presentations of submitted difficult cases that had raised particular diagnostic or legal problems. Topics included concealed births, second impact syndrome, accidents with bicycle helmets, positional asphyxia, and dehydration. The remainder of the day was devoted to 
presentations and discussions on possible mechanisms and neuropathological findings focussing on recent research into sudden and unexpected deaths in epilepsy.

The second day was more eclectic and began with a session on the assessment and dating of bruises. Variability in the histological findings related to the site of biopsy was demonstrated, and interesting data on newer techniques such as molecular biological and optical spectrophotometric methods were elaborated upon. This was followed by a mediolegal review of a recent high profile case of lethal child abuse in Norway, and then presentations on the medical maltreatment of children and on the possible role of $\beta$-amyloid precursor protein in SIDS. The afternoon session included presentations on the historical evolution of our current understanding of SIDS and discussions on neurorespiratory, physiological abnormalities in SIDS involving autoresuscitative gasping, and the role of the brainstem pre-Botzinger complex, as well as the potential role of aquaporins in brain edema in unexpected infant deaths. The investigation of infection-related sudden infant death in Japan was also discussed. The day finished with presentations on the usefulness and deficiencies of death scene reenactments using decedents or dolls, and on the role of animal and mechanical models in clarifying mechanisms of lethal injuries.

The final morning began with a session on autonomic nervous system dysregulation and gene expression in SIDS. The following free paper session included papers on parental responses to and understanding of sudden and unexpected deaths in infancy, brain weight in SIDS, lethal mechanisms in sofa-related deaths, the role of substance $P$ in SIDS, vitamin D deficiency and infant death, inner ear damage and SIDS, stairway injuries in infants, and the manifestations of lethal echovirus infection.

The relatively small number of participants from diverse backgrounds and regions (including Scandinavia, Western Europe, New Zealand, Australia, Japan, and the United
States) at the Soria Moria meetings enables quite free discussion of the presented papers and related issues. In addition, there is ample time in the evenings to meet with fellow professionals and researchers to continue these discussions and to organize future contacts and collaborative studies. The meeting is held at different times throughout the year, and in 2014 is scheduled for June 12th-14th (anne.gunn.winge@fhi.no). The Soria Moria meetings have provided an invaluable forum for over two decades for the discussion and investigation of contentious and evolving concepts in infant and early childhood forensic practice. It is a model that could and should be adopted elsewhere to improve the practice and understanding of this problematic field.

\section{References}

1. Krous HF, Byard RW. Controversies in pediatric forensic pathology. Forensic Sci Med Pathol. 2005;1:9-18.

2. Byard RW. Pediatric forensic pathology-the practice, the prose and the problems. Forensic Sci Med Pathol. 2006;2:135-6.

3. Goudge ST. Inquiry into pediatric forensic pathology in Ontario. 2008. http://www.goudgeinquiry.ca. Accessed June 2013.

4. Byard RW. Unexpected infant death: lessons from the Sally Clark case. Med J Aust. 2004;181:52-4.

5. Krous HF, Langston C. The role of the pediatric pathologist as expert witness in a case of neonatal death. Ped Dev Pathol. 2008;11:200-5.

6. Chadwick D, Krous HF. Irresponsible testimony by medical experts in cases involving the physical abuse and neglect of children. Child Maltreat. 1997;2:313-21.

7. Byard RW. SUDI or "undetermined"-does it matter? Forensic Sci Med Pathol. 2009;5:252-3.

8. Randall BB, Wadee SA, Sens MA, et al. A practical classification schema incorporating consideration of possible asphyxia in cases of sudden unexpected infant death. Forensic Sci Med Pathol. 2009;5:254-60.

9. Baker AM, Crandall L. To hold or not to hold. Forensic Sci Med Pathol. 2009;5:321-3. 